\title{
Marco de Referencia para la Gestión de TI Centrada en la Creación de Valor Compartido, Aplicado a una Propuesta de Formación en Maestría
}

\author{
Information Technology Management Framework \\ FOCUSED ON NATIONAL AND WORLD ORGANIZATIONS. PROPOSAL \\ of a Master's Degree
}

\author{
${ }^{1}$ Andrés Felipe Millán Cifuentes, ${ }^{2}$ Claudio Camilo González \\ ${ }^{1}$ Universidad Nacional Abierta y a Distancia - UNAD, Escuela de Ciencias Básicas, \\ ${ }^{2}$ Tecnología e Ingeniería - ECBTI, Bogotá D.C., Colombia. \\ ${ }^{1}$ andres.millan@unad.edu.co \\ ${ }^{2}$ claudio.gonzalez@unad.edu.co
}

Recibido: 28/04/2014 • Aprobado: 22/06/2014

\section{RESUMEN}

La tecnología de información (TI) es un habilitante para la creación de valor para las organizaciones existentes o nuevas; sin embargo, muchos proyectos que usan $\mathrm{TI}$ fracasan por no lograr alinearse con los objetivos estratégicos de la empresa. Esta realidad ha interesado a la comunidad investigativa que ha trabajado en modelos de referencia para la gestión efectiva de TI orientada a la creación de valor. En años recientes, el concepto de valor ha sido redefinido por autores reconocidos del mundo de los negocios como Porter y Karmer quienes han planteado el enfoque de valor compartido, el cual consiste en que la empresa diseña propuestas de valor para que sus productos y/o servicios impacten positivamente en problemas relevantes de la sociedad. Esta visión reconoce la intersección que hay entre la sociedad y el rendimiento corporativo, orientando a la organización para que sus productos y/o servicios afecten de forma positiva los problemas y necesidades sociales. Esta investigación plantea una propuesta de un marco de referencia para la gestión efectiva de TI orientada a crear valor compartido; igualmente, presenta una aproximación práctica del marco de referencia en el diseño curricular de un programa de maestría en el área de gestión de TI.

Palabras clave: creación de valor compartido, diseño curricular orientado por problemas, gestión de tecnología de información. 


\section{Abstract}

Information technology (IT) is an enabling environment for the creation of value for the new or existing organizations; however, many projects that use IT fail by not achieving aligned with the strategic objectives of the company. This reality has been of interest to the Community research that has worked in reference models for the effective management of IT directed toward the creation of value. In recent years, the concept of value has been redefined by acknowledged authors of the business world as Porter and Karmer, who have proposed that the approach of shared value, which consists in the fact that the company designs proposals of value to their products and/or services, have a positive impact on relevant problems of society. This vision recognizes the intersection between the society and the corporate performance, guiding the organization to their products and/or services affect positively the social problems and needs. This research presents a proposal of a reference framework for the effective management of IT to create shared value; also it presents a practical approach of the framework of reference in the curriculum design of a masters program in the area of IT management.

Keywords: shared value creation, curriculum design problem-oriented, information technology management.

\section{INTRODUCCIÓN}

Investigaciones [1], [2], [3] de comienzos de este siglo muestran que en su mayoría las inversiones realizadas en iniciativas de cambio en los negocios, habilitadas por tecnología de información, están siendo malgastadas o fallan en traer un retorno a la empresa. Por ejemplo, un estudio realizado en 2005 [1] demostró que solo el $8 \%$ del presupuesto gastado en tecnología de información (TI) estaba siendo usado en iniciativas relacionadas en la creación de valor para la empresa.

Esta situación es preocupante al considerar que la nueva visión del liderazgo empresarial está centrada en estrategias de negocio que ofrezcan un ciclo de creación de valor sostenible [4]. Por esta razón, [5] presenta la creación de valor de un negocio como la clave para alcanzar la alineación efectiva entre la estrategia de negocios y la tecnología de información. En consecuencia, [6], [7], [8], [9] han planteado diversos modelos para la creación de valor usando tecnología de información como una estrategia para la transformación organizacional [10].
No obstante, estas investigaciones no han considerado el ciclo de la creación de valor desde la necesidad que tienen las empresas de conectar los negocios con la sociedad, sino como el valor de un negocio como el resultado a corto plazo de una optimización del rendimiento financiero. De esta forma, muchas empresas no satisfacen las necesidades más importantes de sus grupos de interés y hacen caso omiso a las influencias que podrían determinar su éxito empresarial a largo plazo.

Por este motivo, la presente investigación propone un marco de referencia para la creación de valor a partir del modelo de valor compartido de [11] que intenta dar respuesta a esta nueva visión de conectar los negocios y la sociedad. En este mismo sentido, se realizó una aproximación práctica para la implementación del marco propuesto mediante el diseño curricular de un programa académico de nivel de maestría [12] que está siendo ofrecido por la Universidad Nacional Abierta y a Distancia (Unad) con la aprobación del Ministerio de Educación Nacional de Colombia [13]. 
Para realizar este diseño curricular, se utilizó un enfoque en torno a problemas. Este modelo curricular se centra en la identificación de un problema o necesidad social como punto de partida del programa académico [14]. En este caso, el problema validado mediante una investigación rigurosa de la situación en los ámbitos internacional, nacional y regional fue la necesidad en las organizaciones y la sociedad de crear valor compartido hacia sus grupos de interés a través de la gestión efectiva de TI (Tecnología de Información) enfocándose en las necesidades relevantes de empresas, personas y comunidades en las regiones colombianas dentro de un contexto global. Así, el programa de maestría busca contribuir a la formación de talento humano de alto nivel que se ocupe de la gestión de tecnología de información (TI) a partir de este marco de referencia de creación de valor compartido, alcanzando de esta forma uno de los ideales de la disciplina de la tecnología de información (TI) como es "satisfacer las necesidades de los usuarios de una organización o de la sociedad en general a través de la selección, creación, aplicación, integración y administración de las tecnologías computacionales" [15].

Para el desarrollo de este proyecto se propuso una metodología de investigación cualitativa con un enfoque de diseño investigación - acción. Según [16], la investigación - acción es el estudio de una situación social con miras a mejorar la calidad de la acción dentro de ella. [17] plantean la investigación - acción a partir de un proceso de investigación en espiral, en el que se investiga al mismo tiempo que se interviene. En este artículo se presentan los resultados de los dos primeros ciclos de la investigación. Así mismo, está dividido en tres capítulos: el primero aborda el concepto de valor compartido y los trabajos realizados internacionalmente en gestión de $\mathrm{TI}$ centrada en la creación de valor; el segundo capítulo muestra el marco de referencia de gestión de TI propuesto y el último capítulo presenta una propuesta práctica de aplicación del marco de referencia en el diseño curricular de una maestría en gestión de TI.

\section{La Creación de Valor Compartido como EJe de la Gestión Efectiva de TI}

\section{A. El concepto de valor compartido}

Para comprender lo que significa el valor compartido, es importante conocer la evolución histórica del concepto de valor del negocio. En los años sesenta, el valor de un negocio se centraba en maximizar la participación del mercado, pues se asumía que al mantener los costos fijos de una empresa (la mayoría se centraban en productos enfocados en los procesos industriales) e incrementar el volumen de los pedidos, se generaba más valor. En los años ochenta, el enfoque cambió hacia la rentabilidad y las métricas para alcanzar un retorno equitativo centrado en la lógica contable. En los años noventa e inicios de este siglo, el concepto de valor del negocio se enfocó en los retornos financieros ofrecidos por la empresa para sus accionistas, destacándose mediciones como el EVA (valor económico agregado) o MVA (valor agregado del mercado).

En años recientes, el enfoque de valor del negocio ha ido cambiando hacia la creación de valor compartido al reconocer la intersección entre la sociedad y el rendimiento corporativo; esto significa que el valor compartido es más que el cumplimiento de las normas en una empresa, o el desarrollo de programas de responsabilidad social o ayuda voluntaria a la sociedad. Es cuando una organización se focaliza en cómo sus productos y/o servicios y su cadena de valor está afectando importantes problemas o necesidades sociales. [11] proponen un modelo de valor compartido centrado en tres niveles, como se ilustra en la Tabla 1. 
TABLA I

Niveles de Valor Compartido Propuestos por Porter y Otros Autores. [18]

\begin{tabular}{|c|c|c|}
\hline Nivel de valor compartido & Resultados del negocio & Resultados sociales \\
\hline $\begin{array}{l}\text { Reconociendo el producto } \\
\text { y los mercados. } \\
\text { ¿Cómo alcanzar las necesi- } \\
\text { dades insatisfechas puede } \\
\text { mejorar de forma incremental } \\
\text { los ingresos y beneficios de un } \\
\text { negocio? }\end{array}$ & $\begin{array}{l}\text { Incremento en los ingresos. } \\
\text { Incremento en la participa- } \\
\text { ción del mercado. } \\
\text { Incremento del crecimiento } \\
\text { del mercado. } \\
\text { Mejora en la rentabilidad. }\end{array}$ & $\begin{array}{l}\text { Mejora el estado de salud del pacien- } \\
\text { te. } \\
\text { Mejora la nutrición. } \\
\text { Mejora la educación. } \\
\text { Reduce la ".huella de carbono". }\end{array}$ \\
\hline $\begin{array}{l}\text { Redefiniendo la productivi- } \\
\text { dad en la cadena de valor. } \\
\text { ¿Cómo mejorar la gestión de } \\
\text { las operaciones internas para } \\
\text { incrementar la productividad y } \\
\text { reducir los riesgos? }\end{array}$ & $\begin{array}{l}\text { Mejora en la productividad. } \\
\text { Reducción de costos opera- } \\
\text { cionales o logísticos. } \\
\text { Mejora la calidad. } \\
\text { Provee más seguridad. } \\
\text { Mejora la rentabilidad. }\end{array}$ & $\begin{array}{l}\text { Reduce uso de energía } \\
\text { Reduce uso de agua. } \\
\text { Reduce materia prima. } \\
\text { Mejora habilidades laborales. } \\
\text { Mejora ingresos a los empleados. }\end{array}$ \\
\hline
\end{tabular}

\section{B. Trabajos en valor del negocio de tecnología de información}

En 1987, Robert Solow, premio nobel de economía, inició un debate muy conocido denominado la "paradoja de la productividad" cuando dijo: "la era de la computación está en todas partes, excepto en las estadísticas de productividad" [19], [20] concluyó que esta paradoja existe por la combinación de varios factores, siendo los más relevantes: los errores de medición tanto en el capital y los resultados de $\mathrm{Tl}$, la diferencia en el tiempo entre la inversión de TI y las ganancias de productividad y la falta de buenas prácticas de gestión que permiten descubrir las oportunidades potenciales de la tecnología de información.

Pero fueron [6] quienes mostraron que la realización de valor mediante TI produce entradas complementarias y capital organizacional, aspectos que se relacionan con la productividad empresarial. Esta investigación reforzó las conclusiones parciales a las que se había llegado en otros estudios que demostraban que la inversión de TI combinada con el rediseño organizacional [21] y la mejora en las prácticas y procesos de negocios producían efectos positivos en algunos indicadores de productividad [22].
Este trabajo investigativo ha demostrado que más allá de las métricas económicas, el problema principal está en la transformación organizacional centrada en TI [10]. De allí, que varios autores propongan modelos de creación de valor usando TI como una estrategia para la transformación organizacional. Entre los modelos de referencia más reconocidos están:

- Modelo DeLone y McLean [7] o Modelo D\&M: se focaliza en la calidad de los sistemas y de la información para lograr un uso efectivo de los sistemas de información que garantice la satisfacción del usuario, lo cual produce un impacto individual que finalmente genera un impacto organizacional.

- Modelo RBV [8]: centrado en valor mediante $\mathrm{Tl}$; se concentra en vistas basadas en recursos. Estos recursos se pueden agrupar en tres niveles: la propia empresa, el ambiente competitivo y el macroambiente.

- Modelo de Rivard y otros [9]: integra la vista en recursos con la estrategia corporativa. 
- Modelo de Gregor y otros [10]: reconoce la necesidad de un enfoque cíclico para interpretar el valor de TI para el negocio. Destaca la relación bidireccional que hay entre la influencia organizacional y del sector de la industria con el valor de TI para el negocio y viceversa, todo esto dentro de las influencias de un macroambiente.

\section{Marco de Referencia Parala Creación de Valor Compartido con Tecnología DE INFORMACIÓN (TI)}

A partir de los modelos mencionados en el apartado anterior, esta propuesta trae los siguientes conceptos para la construcción del marco de referencia propuesto:

- El marco de referencia se centra en la creación de valor como tendencia en todos los modelos propuestos.

- La perspectiva de vistas por recursos propuesta por los modelos de RBV y de Rivard y otros se ha homologado a la visión actual de capacidades del negocio, gente y TI propias de la práctica de arquitectura empresarial.

- El enfoque cíclico propuesto por Gregor y otros demuestra que la naturaleza de la creación de valor no es lineal sino iterativa y contributiva.

En sintonía con la revisión realizada, la pregunta relevante del equipo investigador fue: ¿Cómo podemos propiciar una relación bidireccional entre la influencia organizacional, el efecto de la industria y el macroambiente para crear valor a partir de TI? La respuesta más acorde en este sentido es el concepto de valor compartido, pues hace una intersección entre la empresa y la Sociedad. En este sentido, [18] proponen un proceso de 4 etapas que incluye:

- Identificar los asuntos sociales a los que se debe apuntar.Hacer un caso de negocio.Hacer seguimiento a los progresos. Medir los resultados y los insight para encontrar nuevo valor.

El equipo investigador consideró que era posible integrar el proceso de creación de valor compartido con una visión enfocada en la práctica de arquitectura empresarial centrada en responder a tres preguntas generadoras del marco de referencia: ¿por qué una organización desea crear valor para sus dueños y para la sociedad a través de la gestión efectiva de TI?, ¿qué capacidades de negocio y de TI debe tener una organización (blueprint) para crear valor a la empresa y a la sociedad? y ¿cómo implementar ambientes de TI innovadores para alcanzar la propuesta de valor para la empresa y la sociedad? Este enfoque se estructuró en un marco de referencia cíclico de creación de valor compartido usando TI para lograr una actuación local (en este caso, las comunidades y regiones colombianas) desde una perspectiva global que se presenta en la tabla 2. Este marco de referencia utiliza varias herramientas metodológicas probadas como Lean Startup [23], la generación de modelos de negocios [24], el diseño de propuestas de valor [25] y la práctica de arquitectura de negocios [33], entre otras. 
TABLA II

Marco de Referencia para Gestión Efectiva de TI para Crear Valor Compartido

\begin{tabular}{|c|c|c|}
\hline \multicolumn{3}{|c|}{$\begin{array}{l}\text { Marco de Referencia para la gestión efectiva de TI } \\
\text { para crear valor compartido }\end{array}$} \\
\hline $\begin{array}{l}\text { Proceso de medición de valor } \\
\text { compartido } \\
\text { Portery otros autores [19] }\end{array}$ & $\begin{array}{l}\text { Componentes del Marco de } \\
\text { referencia }\end{array}$ & Metodología propuesta \\
\hline $\begin{array}{l}\text { 1. Identificar los asuntos sociales } \\
\text { a los que apuntar }\end{array}$ & $\begin{array}{l}\text { 1. Diseño de la propuesta de } \\
\text { valor compartido }\end{array}$ & $\begin{array}{l}\text { 1. Identificar metas y objetivos a } \\
\text { partir de un asunto social } \\
\text { 2. Entender la motivación del negocio } \\
\text { 3. Construir propuestas de valor } \\
\text { compartido } \\
\text { 4. Prototipar modelos estratégicos de } \\
\text { negocios } \\
\text { 5. Definir componentes de la cadena } \\
\text { de valor }\end{array}$ \\
\hline 2. Hacer un caso de negocio & $\begin{array}{l}\text { 2. Blueprint de capacidades } \\
\text { del negocio y de TI de la } \\
\text { empresa }\end{array}$ & $\begin{array}{l}\text { 6. Definir capacidades del negocio y } \\
\text { de TI } \\
\text { 7. Asegurar la madurez de las } \\
\text { capacidades (AS-IS) } \\
\text { 8. Definir estado futuro de la } \\
\text { maduraz de la capacidades (TO-BE) } \\
\text { 9. Oportunidades y soluciones } \\
\text { 10. Plan de la Transición }\end{array}$ \\
\hline $\begin{array}{l}\text { 3. Hacer un seguimiento a los } \\
\text { progresos }\end{array}$ & & $\begin{array}{l}\text { 11. Gestión ágil de proyectos } \\
\text { 12. Construir prototipos del producto } \\
\text { y/o servicio } \\
\text { 13. Análisis y métricas de los }\end{array}$ \\
\hline $\begin{array}{l}\text { 4. Medir los resultados y los } \\
\text { insights para encontrar nuevo } \\
\text { valor }\end{array}$ & $\begin{array}{c}\text { ambiente innovador basado } \\
\text { en TI }\end{array}$ & $\begin{array}{l}\text { 14. Optimizar el modelo de negocios } \\
\text { 15. Gobierno corporativo } \\
\text { 16. Aprovisionamiento y soporte del } \\
\text { producto y/ servicio } \\
\text { 17. Gestión del cambio }\end{array}$ \\
\hline
\end{tabular}

\section{Propuesta de Aplicación Práctica: Programa Académico de Maestría en Gestión de TI en la Unad}

\section{A. Marco contextual del Programa Académico}

La formación de talento humano a nivel de maestría y doctorado se considera como elemento nuclear en procesos de desarrollo competitivo y sostenible de comunidades, instituciones y naciones. Esto en razón a que las capacidades de una sociedad para generar conocimiento e innovaciones están estrechamente ligadas a las capacidades desarrolladas por comunidades de personas para abordar problemas $u$ oportunidades que, a partir de procesos de investigación, generen nuevos enfoques teóricos o desarrollos tecnológicos.
En el contexto internacional, la Organización de los Estados Iberoamericanos, OEI, [27] establece dentro de los retos de los países iberoamericanos uno denominado "Fortalecer la Capacidad Científica y Tecnológica", en el que se articulan elementos relacionados con: capacidades para cualificación de científicos y expertos, apropiación y adaptación de conocimiento, transferencia de conocimiento, vinculación a redes, cooperación internacional, identificación de problemas/oportunidades, entre otros, entendiendo que "Gestionar estas posibilidades con un sentido estratégico, sobre la base de un profundo conocimiento de las necesidades locales, es un aspecto de la madurez científica y tecnológica". En forma paralela, el World Economic Forum, en el GCI [28], establece dos indicadores relacionados con la formación pos gradual: Tertiary education enrollment y Availability of scientists and engineers. Ello demuestra la importancia que le reconocen organismos 
multilaterales y países desarrollados a la cualificación de alto nivel, como elemento clave para la economía basada en el conocimiento.

En el contexto nacional, este nivel de formación parece que, a juzgar por análisis diversos, no ha recibido el mismo apoyo que el destinado a la formación de grado [29]. De acuerdo con el Sistema Nacional de Información de la Educación Superior, Colombia cuenta con 5.180 programas de posgrado activos, de los cuales el $68 \%$ son de especialización, y no están orientados a desarrollar capacidades de investigación del talento humano, sino a perfeccionar competencias profesionales. Desde las lógicas del aporte de la educación superior en el desarrollo regional, el Índice Departamental de Competitividad [30] señala enormes desigualdades en la graduación a nivel de posgrado, tal como se aprecia en la Fig. 1.

De acuerdo con la Fig. 1, la metodología de medición asigna el máximo puntaje al departa- mento que mayor población graduada tiene. Se aprecia que el máximo nivel lo tiene Santander, mientras que de los restantes solo Bogotá supera el nivel de mitad del rango establecido. Considerando otros indicadores de desarrollo, ni siquiera aquellos departamentos que concentran los mayores aportes al PIB, presentan niveles competitivos en formación de posgrado; se esperaría que, en consecuencia, los procesos de Ciencia Tecnología e Innovación tengan limitaciones en cuanto a talento humano disponible con las capacidades requeridas para garantizar competitividad global.

Considerando el contexto de la sociedad de la información y el conocimiento y la respuesta de la academia para responder a los retos que se le presentan al país, se analiza la oferta de programas de formación de alto nivel. En SNIES se registran, en el área de Ingeniería, Arquitectura, Urbanismo y afines, dos núcleos básicos de conocimiento: Ingeniería de Sistemas, Telemática y afines, e Ingeniería Electrónica, Telecomunicaciones y afines.

\section{Pilar 7- Indicador Graduados de Posgrado Puntaje Ponderado \\ (0 a 10)}

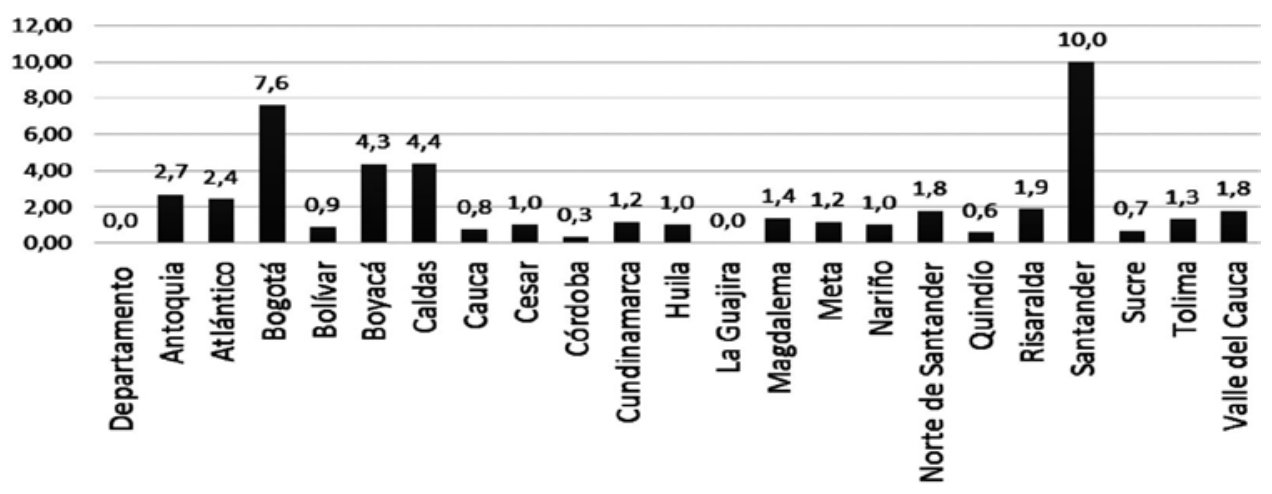

Fig. 1 Valoración del nivel de graduados en formación de posgrado [30]

De acuerdo con la Fig. 1, la metodología de medición asigna el máximo puntaje al departamento que mayor población graduada tiene. Se aprecia que el máximo nivel lo tiene Santander, mientras que de los restantes solo Bogotá supera el nivel de mitad del rango establecido. Considerando otros indicadores de desarrollo, ni siquiera aquellos departamentos que concentran los mayores aportes al PIB, presentan niveles competitivos en formación de posgrado; se esperaría que, en consecuencia, los procesos de Ciencia Tecnología e Innovación tengan limitaciones en cuanto a talento humano disponible con las capacidades requeridas para garantizar competitividad global. 
Considerando el contexto de la sociedad de la información y el conocimiento y la respuesta de la academia para responder a los retos que se le presentan al país, se analiza la oferta de programas de formación de alto nivel. En SNIES se registran, en el área de Ingeniería, Arquitectura, Urbanismo y afines, dos núcleos básicos de conocimiento: Ingeniería de Sistemas, Telemática y afines, e Ingeniería Electrónica, Telecomunicaciones y afines.

Estos son los únicos núcleos que están directamente relacionados con la formación de talento humano de alto nivel en actividades de ciencia, tecnología e innovación, que aporten a la incursión del país en la economía del conocimiento basado en desarrollos e innovaciones en el campo de la Tecnología de Información (TI). En la Tabla 3 se muestran los programas que se desarrollan en estos dos núcleos, de acuerdo con el SNIES.

TABLA III

Cantidad de Programas de Doctorado y Maestría en Núcleos Básicos de Conocimiento Relacionados CON TECNOLOGía de INFORMACIÓN. [31]

\begin{tabular}{|l|c|c|}
\hline $\begin{array}{c}\text { Nivel de } \\
\text { formación }\end{array}$ & $\begin{array}{c}\text { Número de } \\
\text { programas }\end{array}$ & $\begin{array}{c}\text { Porcentaje de } \\
\text { participación frente al } \\
\text { total de programas de } \\
\text { maestría y doctorado }\end{array}$ \\
\hline Maestría & 67 & $5 \%$ \\
\hline Doctorado & 10 & $4 \%$ \\
\hline
\end{tabular}

En consecuencia, solo el $5 \%$ de los programas de maestría y el $4 \%$ de los programas de doctorado del país están orientados a formar talento humano para desarrollar investigación en el campo de Tecnología de Información (TI). Estos porcentajes se pueden considerar muy bajos en relación con las propuestas de formación en otras áreas de conocimiento, si se considera que las tecnologías de información son denominadas núcleo fundamental de la sociedad de la información y la economía del conocimiento.
De acuerdo con el SNIES [31, el número de graduados en los últimos cuatro (4) años como se muestra en la Tabla 4, es de veintitrés (23) doctores y mil seiscientos veintitrés (1.623) magister, en programas de campos de conocimiento relacionados con TI. Según estudio de [32], citado por [33], se identifica que en el talento humano vinculado al sector software y servicios asociados "los niveles de formación académica más avanzados (doctor y máster) son los de menor peso relativo: solo 51 empleados. El $0.2 \%$, tiene un grado de formación de doctor y 288 empleados, el 1\%, de maestría". De hecho, el mismo estudio concluye que una oferta muy baja de capital humano con formación de alto nivel representa una debilidad para el desarrollo del sector.

TABLA IV

Graduados en Programas de Maestría Y DOCTORADO 2010-2013. [31]

\begin{tabular}{|l|c|c|c|c|}
\hline $\begin{array}{c}\text { Nivel de } \\
\text { Formación }\end{array}$ & 2013 & 2012 & 2011 & $\mathbf{2 0 1 0}$ \\
\hline Maestría & 354 & 435 & 388 & 446 \\
\hline Doctorado & 12 & 7 & 3 & 1 \\
\hline
\end{tabular}

Los análisis realizados por [32] establecen que el perfil de maestría es requerido para cargos como arquitecto de software senior, director de proyecto, gerente de desarrollo, gerente de operaciones/producción y líder consultor senior. De igual forma, estos cargos están asociados a tres variables: son los más difíciles de encontrar en el mercado laboral, requieren los mayores niveles de cualificación (predominando el de posgrado) y demandan certificaciones en ITIL y PMP.

En relación con la formación de alto nivel orientada a desempeños profesionales que requieren competencias de liderazgo, las recomendaciones consignadas en estudio realizado por [34] sobresale el relacionado con la formación en un nuevo enfoque en las 
universidades: "deberán ofrecer programas avanzados, programas interdisciplinarios y programas de maestrías integradas en el campo de las tecnologías de la información para crear una nueva generación de líderes". Así mismo, interpretando el punto de vista gubernamental, afirma que "Colombia debe desarrollar talento en Big Data, movilidad, analítica, ciber-seguridad, arquitectura de TI, gobierno en línea y administración de proyectos".

\section{B. Maestría en gestión de tecnología de in- formación de la Unad.}

Considerando el contexto internacional, nacional y regional, así como el marco de referencia para la gestión efectiva explicado en el apartado anterior, la Unad propone el programa académico de la Maestría en Gestión de Tecnología de Información (TI) que se basa en el diseño curricular en torno a problemas y por competencias. El núcleo problémico se entiende como la unidad integradora que posibilita pensar en procesos de investigación alrededor de objetos de transformación y sugiere estrategias metodológicas que garantizan la síntesis creativa entre la teoría y la práctica. El núcleo problémico es un problema, una necesidad, una oportunidad o un vacío en el conocimiento que aglutina diferentes disciplinas, permitiendo un abordaje integral, un tipo de mediación pedagógica y una propuesta didáctica específica, con el fin de contribuir a la formación del estudiante y generar conocimiento pertinente tanto para los contextos sociales como para las mismas disciplinas. De otra parte, la Unad recoge el concepto de Núcleo Integrador de Problema (NIP), como metodología válida para el diseño curricular.
La integración es un principio pedagógico que promueve la vinculación permanente de todos los conocimientos de las áreas disciplinarías o campos culturales que se trabajan en la universidad, entre sí y con el mundo de la vida, buscando ante todo hacer verdad que el saber tiene sentido [35]. Los núcleos integradores son una manera de concretar las propuestas conceptuales sobre la conversación para la formación, las competencias como expresiones de la totalidad de la persona y la complejidad del mundo y de la sociedad.

En este contexto, el núcleo integrador de problema y los núcleos problémicos planteados para el Programa de Maestría en Gestión de TI de la Unad, surgen como resultado de una rigurosa revisión del estado actual de la profesión y de la identificación de las necesidades y competencias que debe satisfacer el candidato a magister, a través de búsqueda documental de información secundaria, y consulta realizada a empresarios, que permitieron identificar necesidades y características homogéneas del egresado de este programa.

Es importante resaltar, que los núcleos problémicos se identificaron con base en manifestaciones que evidencian el problema, y unas preguntas generadoras que surgen como artificio integrador y cuya respuesta orienta la aplicación de la profesión en la solución de dichos problemas desde la gestión de TI. La Fig. 2 muestra la alineación entre el marco de referencia de gestión de TI para crear valor compartido y los núcleos problémicos del programa académico. La Tabla 5 detalla el núcleo integrador del problema (NIP) del programa. 


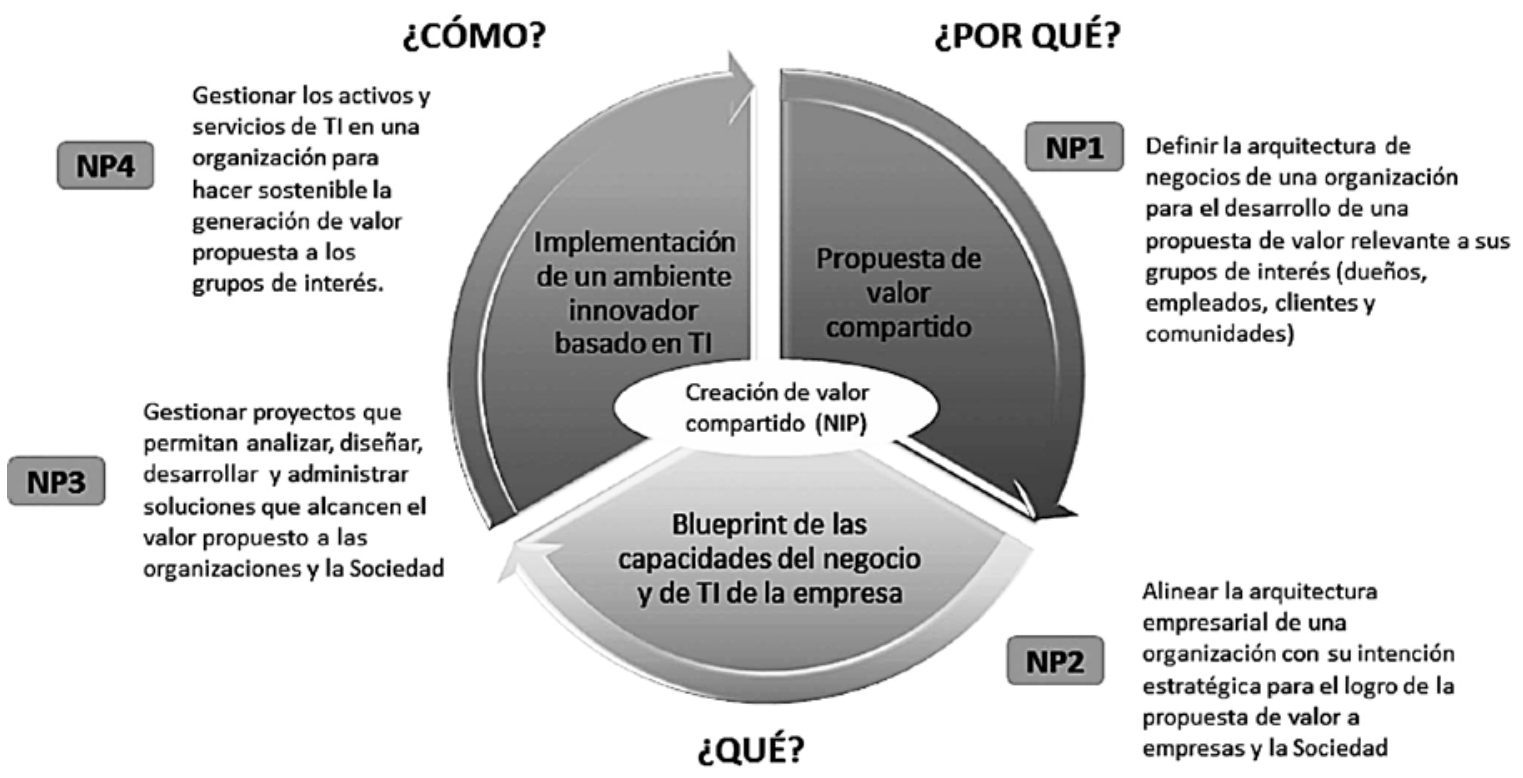

Fig. 2. Relación del núcleo integrador del problema (NIP) y los núcleos problémicos con el Marco de referencia de gestión de TI para crear valor compartido. [12]

TABLA V.

Núcleo Integrador de Problema (Nip) de la Maestría en Gestión de TI de la Unad. [12]

\section{NIP - Núcleo Integrador de Problema}

La necesidad en las organizaciones y la Sociedad de crear valor compartido hacia sus grupos de interés a través de la gestión efectiva de TI (Tecnología de Información) enfocándose en los problemas relevantes de empresas, personas y comunidades en las regiones colombianas dentro de un contexto global.

\begin{tabular}{|c|c|c|c|}
\hline Manifestaciones & & & Competencias \\
\hline $\begin{array}{l}\text { Baja adopción de una } \\
\text { gestión efectiva de TI } \\
\text { en las microempresas } \\
\text { y pequeñas empresas } \\
\text { colombianas. } \\
\text { La gecesidad } \\
\text { de gestionar las } \\
\text { herramientas y } \\
\text { tecnologías de } \\
\text { información desde } \\
\text { una perspectiva } \\
\text { estratégica y no } \\
\text { meramente operativa } \\
\text { en las organizaciones. }\end{array}$ & $\begin{array}{l}\text { ¿Por qué una or- } \\
\text { ganización desea } \\
\text { crear valor para } \\
\text { sus dueños y para } \\
\text { la Sociedad a tra- } \\
\text { vés de la gestión } \\
\text { efectiva de Tl? }\end{array}$ & $\begin{array}{l}\text { NP1 - Definir la arquitec- } \\
\text { tura de negocios de una } \\
\text { organización para el de- } \\
\text { sarrollo de una propuesta } \\
\text { de valor relevante a sus } \\
\text { grupos de interés (due- } \\
\text { ños, empleados, clientes } \\
\text { y comunidades). }\end{array}$ & $\begin{array}{l}\text { CMGTI1: capacidad de identificar } \\
\text { y explotar las oportunidades que } \\
\text { ofrecen diversas tecnologías, } \\
\text { servicios y productos de TI para } \\
\text { el beneficio de personas, grupos } \\
\text { y organizaciones en diferentes } \\
\text { dominios (salud, gobierno, } \\
\text { educación, entre otros). } \\
\text { CMGTI2: capacidad de proponer } \\
\text { modelos estratégicos de negocios } \\
\text { centrados en el desarrollo de una } \\
\text { propuesta de valor hacia sus } \\
\text { grupos de interés. }\end{array}$ \\
\hline $\begin{array}{l}\text { Falta de aplicaciones } \\
\text { y contenidos locales } \\
\text { que hagan que los } \\
\text { ciudadanos y las } \\
\text { empresas encuentren } \\
\text { información relevante } \\
\text { y útil para su } \\
\text { competitividad. }\end{array}$ & $\begin{array}{l}\text { ¿Qué capacidades } \\
\text { de negocio y de TI } \\
\text { debe tener una or- } \\
\text { ganización (blue- } \\
\text { print) para crear } \\
\text { valor a la empresa } \\
\text { y a la sociedad? }\end{array}$ & $\begin{array}{l}\text { NP2 - Alinear la arquitec- } \\
\text { tura empresarial de una } \\
\text { organización con su in- } \\
\text { tención estratégica para } \\
\text { el logro de la propuesta } \\
\text { de valor a la empresa y la } \\
\text { sociedad. }\end{array}$ & $\begin{array}{l}\text { CMGTI3: capacidad de alinear, } \\
\text { elaborar y gobernar la arquitectura } \\
\text { empresarial (capacidades de } \\
\text { gente, procesos y herramientas) } \\
\text { de una organización para alcanzar } \\
\text { su propuesta de valor hacia sus } \\
\text { grupos de interés. }\end{array}$ \\
\hline
\end{tabular}




\begin{tabular}{|c|c|c|c|}
\hline Manifestaciones & $\begin{array}{l}\text { Pregunta } \\
\text { generadora }\end{array}$ & $\begin{array}{l}\text { NP - Núcleo } \\
\text { problémico }\end{array}$ & Competencias \\
\hline $\begin{array}{l}\text { Falta de recurso altamen- } \\
\text { te capacitado en TI para el } \\
\text { desarrollo económico de } \\
\text { las regiones } \\
\text { y el país. } \\
\text { Falta de una infraestruc- } \\
\text { tura de TI segura y de } \\
\text { alta calidad en algunas }\end{array}$ & $\begin{array}{l}\text { ¿Cómo imple- } \\
\text { mentar ambien- } \\
\text { tes de TI inno- } \\
\text { vadores para }\end{array}$ & $\begin{array}{l}\text { NP3 - Gestionar proyec- } \\
\text { tos que permitan analizar, } \\
\text { diseñar, desarrollar y ad- } \\
\text { ministrar soluciones que } \\
\text { alcancen el valor propues- } \\
\text { to a las organizaciones y } \\
\text { la sociedad. }\end{array}$ & $\begin{array}{l}\text { CMGT4: capacidad para gestio- } \\
\text { nar proyectos innovadores que } \\
\text { aseguren el valor propuesto a la } \\
\text { organización y a la sociedad. }\end{array}$ \\
\hline $\begin{array}{l}\text { regıones del pais. } \\
\text { Una industria de TI en } \\
\text { crecimiento, pero con } \\
\text { deficiencias para con- } \\
\text { vertirse en un sector de } \\
\text { clase mundial para Co- } \\
\text { lombia. }\end{array}$ & $\begin{array}{l}\text { puesta de valor } \\
\text { y la sociedad? }\end{array}$ & $\begin{array}{l}\text { NP4- Gestionar los acti- } \\
\text { vos y servicios de TI en } \\
\text { una organización para } \\
\text { hacer sostenible la gene- } \\
\text { ración de valor propuesta } \\
\text { a los grupos de interés. }\end{array}$ & $\begin{array}{l}\text { CMGTI5: capacidad para gestio- } \\
\text { nar los activos y servicios de TI } \\
\text { estableciendo un proceso de me- } \\
\text { jora continua para la generación } \\
\text { de valor dentro de la organiza- } \\
\text { ción. }\end{array}$ \\
\hline
\end{tabular}

Pasar de los núcleos problémicos a las competencias implica comprometer una intencionalidad formativa en términos de las competencias que se considera deben ser desarrolladas por un ser humano, para desempeñarse efectivamente en un campo específico. La Fig. 3 muestra la relación de las competencias específicas del programa, con el marco de referencia propuesto.

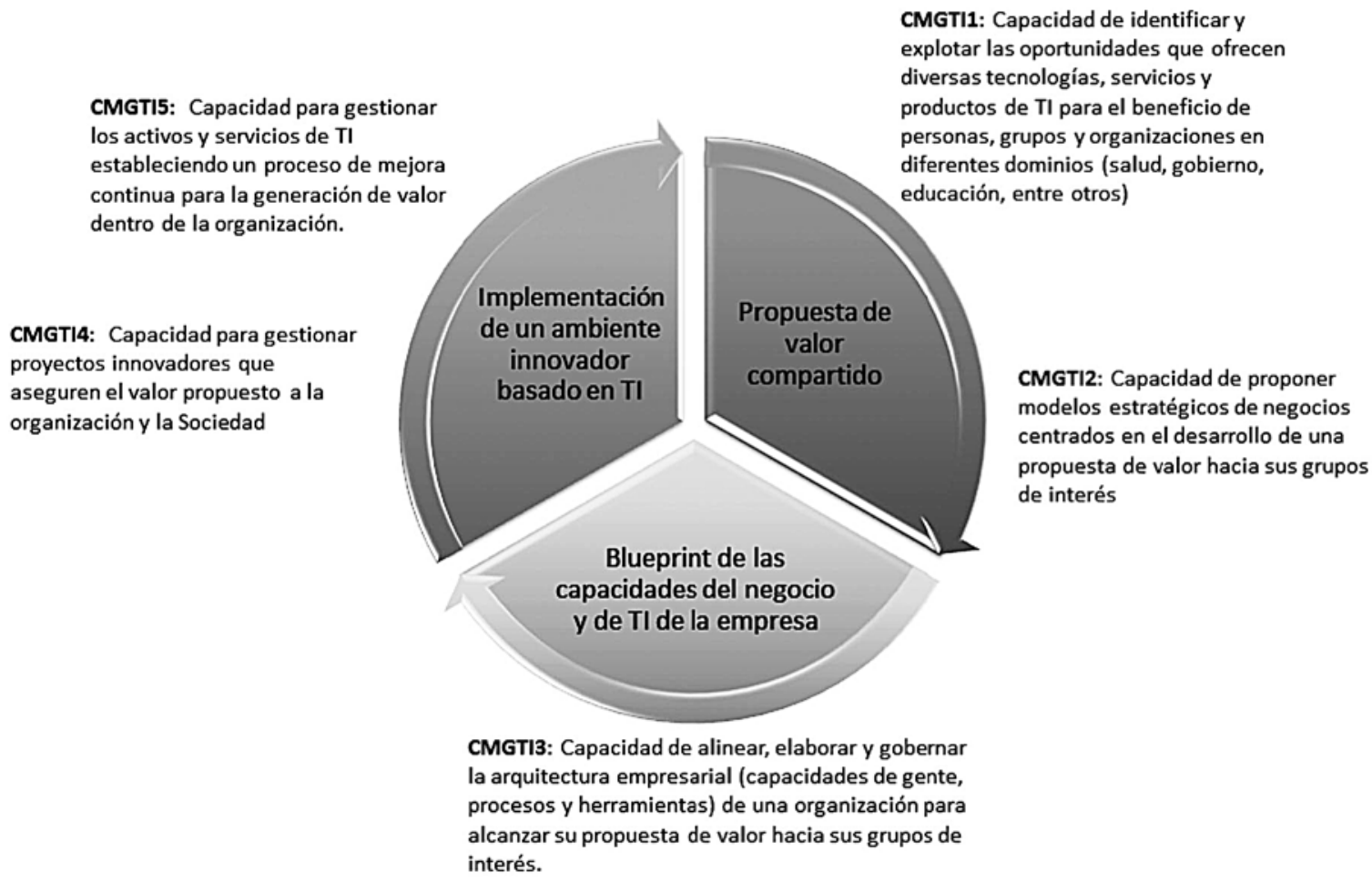

Fig. 3 Marco de referencia para la gestión efectiva de TI vs Competencias Específicas del programa. [12] 


\section{Conclusiones}

El presente trabajo ha permitido alcanzar dos conclusiones relevantes: la primera, de interés para la comunidad científica, relacionada con el desarrollo de un marco de referencia para la gestión de TI orientado a la creación de valor compartido. Este documento presenta varios referentes internacionales que han enfocado sus propuestas de marco de referencia para gestión de $\mathrm{TI}$ en la creación de valor; sin embargo, se ha encontrado una brecha en lo que [10] identifican como la bidireccionalidad de la transformación organizacional mediante $\mathrm{TI}$, que implica un proceso cíclico que debe incluir la influencia de la empresa, el efecto de la industria y el macro ambiente. Considerando este problema

abierto, el equipo investigador propuso como eje articulador del marco de referencia objetivo el concepto de valor compartido propuesto por [36], pues este intenta explicar la creación de valor desde la conexión entre los negocios y la sociedad. Para articular el marco de referencia se ha fundamentado la propuesta en los trabajos adelantados por la firma consultora [37] en el concepto de creación de valor compartido.

La segunda conclusión relevante de esta investigación es la necesidad de formar talento humano para una gestión efectiva de TI que siga un marco de referencia que pueda dar respuesta a los problemas sociales, tecnológicos, ambientales y económicos desde una visión holística de las capacidades de la empresa y de TI para la creación de valor compartido. Esta visión permitió al equipo investigador proponer el diseño de un programa académico de maestría que tiene como base el marco de referencia propuesto y que ya ha sido aprobado por el Ministerio de Educación Nacional de Colombia [13] para ser impartido por la Universidad Nacional Abierta y a Distancia (Unad) en una modalidad virtual.
Como parte del trabajo futuro, se ha planeado realizar un proyecto el cual permita construir una metodología que facilite la aplicación del marco de referencia en las empresas. De la misma manera, en la hoja de ruta de esta investigación, siguiendo la metodología de investigación - acción, se ha propuesto un estudio de la implementación del programa de maestría en los estudiantes y en los proyectos de grado ejecutados por ellos en las regiones colombianas.

\section{Referencias}

[1] Butler Group, "Measuring IT Costs and Value." Londres: DataMonitor Group, 2005.

[2] N. Huber, Gartner: Firms waste £351bn Each Year on III-conceived IT Projects. London: Computerweekly. com, 2002.

[3] R. Cook, (2007), How to Spot a Failing Project. ClO Magazine. Recuperado de: http://www.cio.com/article/2438424/project-management/how-to-spot-afailing-project.html.

[4] Accenture Committee Encouring Corporate Philanthrophy, Business at its Best: Driving Sustainable Value Creation: Five Imperatives for Corporate CEOs. Nueva York: Accenture. Recuperado de: http:// csis.org/files/attachments/111017_CECP_Accenture. pdf, 2011.

[5] A. Bathia, Value Creation: Linking Information Technology and Business Strategy. Dallas: Brown Books Publishing Group, 2012.

[6] E. Brynjolfsson, L. Hitt, Computer productivity: firmlevel evidence. MIT Sloan Working Paper No 421001. Boston: MIT Sloan, 2003.

[7] W. DeLone, E. McLean, "The DeLone and McLean model of information system success: a ten-year update." Journal Management Information Systems, 19 (4), 9-30, 2003.

[8] N. Melville, K. Kraemer, V. Gurbuxani, "Review: information technology and organizational performance: an integrative model of IT business value." MIS Q. 28 (2), 283-322, 2004.

[9] S. Rivard, L. Raymond, D. Verreault, "Resource-based view and competitive strategy: an integrated model of the contribution of information technology to firm performance." Journal Management Information Systems, 15(1), 29-50, 2006.

[10] S. Gregor, S., M. Martin, Fernandez, S. Stern, M. Vitale, M., "The transformational dimension in the realization of business value from information technology". Journal of Strategic Information Systems, 15, 249-270, 2006. 
[11] M. Porter y M. Kramer, "Creating Shared Value." Harvard Business Review. Enero-Febrero de 2011, 6277, 2011.

[12] UNAD, Documento Maestría en Gestión de TI. Bogotá DC: Universidad Nacional Abierta y a Distancia, 2014.

[13] Ministerio de Educación Nacional de Colombia, ResoIución 10107. Bogotá DC: Ministerio de Educación Nacional de Colombia, 2015. http://snies.mineducacion. gov.co/consultasnies/verPrograma?codigo $=104690$.

[14] J. Barrel, El aprendizaje basado en problemas: un enfoque investigativo. Buenos Aires: Manantial, 1999.

[15] IEEE Computer Society, Information Technology 2008 - Curriculum Guidelines for Undergraduate Degree Programs in Information Technology. Nueva York: IEEE y ACM, 2008.

[16] J. Elliot, Action research for educational change. Buckingham: Open University Press, 1991.

[17] O.G. León, I. Montero, Métodos de investigación en psicología y educación ( $3^{\underline{a}}$ ed.). Madrid: MacGrawHill Interamericana, 2003.

[18] M. Porter, G. Hills, M. Pfitzer, S. Patscheke, S. y E. Hawkins, Measuring Shared Value. Boston: FSG, 2012.

[19] R. Solow, "We'd better watch out". New York Times Review. Julio, p.36 New York: New York Times, 1987.

[20] E. Brynjolfsson, "The productivity paradox of information technology". Commun. ACM , 35 (12), 66-77. Nueva York: ACM, 1993.

[21] R. Murnane, F. Levy, Tecnological change, computer and skills demands: evidence from back office operations of a large bank. Mimeo, NBER Economic Research Labor Workshop, 1999.

[22] E. Brynjolfsson, L. Hitt, L., "Beyond computation: Information Technology, organizational transformation and business performance". J. Econom. Perspect. 14 (4), 23-28. Nashville: AEA, 2000.

[23] E. Ries, The Lean Startup: How Today's Enterpreneurs use continuous innovation to create radically successful businesses. Nueva York: Crown Business, 2011.

[24] A. Osterwalder y. Pigneur, Business Model Generation. Haboken, New Yersey: Wiley, 2010.

[25] Osterwalder et al., Value Proposition Design. Haboken, New Yersey: Wiley, 2014.
[26] Martin, C. (2014), Discovering Business Architecture. Enterprise Architects. Recuperado de: http://enterprisearchitects.com/webinar-discovering-businessarchitecture/\#DBASlides.

[27] M. Albornoz, (Coordinador), Ciencia, Tecnología e Innovación Para el Desarrollo y la Cohesión Social. Un programa iberoamericano en la década de los bicentenarios. Madrid: OEI, 2014.

[28] Schwab, Klaus (2014). The Global Competitiveness Report 2014-2015. World Economic Forum. Recuperado de http://www3.weforum.org/docs/WEF_GlobalCompetitivenessReport_2014-15.pdf.

[29] H. Jaramillo, H. "La formación de posgrado en Colombia: maestrías y doctorados". Revista iberoamericana de ciencia tecnología y sociedad [online]. Vol.5, n.13, pp. 131-155, 2009. Recuperado de: http:// www.scielo.org.ar/pdf/cts/v5n13/v5n13a08.pdf

[30] Consejo Privado de la Competitividad, Universidad del Rosario, Índice Departamental de Competitividad 2014. Bogotá D.C: Universidad del Rosario, 2014.

[31] SNIES, Sistema de Información de la Educación Superior. Colombia. http://www.mineducacion. gov.co/sistemasdeinformacion/1735/w3-propertyname-2672.html. Fecha de consulta: marzo 20 de 2015.

[32] FEDESOFT, Estudio de Salarios y Profesionales del Sector de Software y TI de Colombia 2012. Informe sectorial de la industria de software y servicios asociados de Colombia. Bogotá DC: FEDESOFT, 2012.

[33] MINTIC, Visión Estratégica del Sector. Plan de Mercadeo y Ventas de Software y Servicios Asociados. Bogotá DC: MINTIC, 2013.

[34] MINTIC, Infosys y Universidad EAFIT, Brecha de Talento Digital. Contrato Estatal para el desarrollo de actividades científicas y tecnológicas № 000786 de 2013 entre el fondo de Tecnologías de la Información y La Universidad EAFIT. Bogotá DC: MINTIC, 2012.

[35] R. Montero, Currículo Problémico. En: Congreso Pedagógico Unadista. Bogotá DC: UNAD, 2011.

[36] M. Porter, M. Kramer, M., "The link between competitive advantage and corporate social responsibility". Harvard Business Review. 2006.

[37] FSG (2016), Portal web de la organización FSG. Recuperado de: http://www.fsg.org/. 
